\title{
New Results on DLA Systems: Statistics
}

\author{
Sandhya Rao ${ }^{1}$, David Turnshek ${ }^{1}$, Eric Monier ${ }^{2}$ and Gendith Sardane ${ }^{1}$ \\ ${ }^{1}$ University of Pittsburgh and the Pittsburgh Particle Physics, Astrophysics and Cosmology \\ Center (PITT PACC), Pittsburgh, PA 15260, USA \\ email: srao@pitt.edu \\ 2 The College at Brockport, State University of New York, Brockport, NY 14420, USA
}

\begin{abstract}
The damped Lyman- $\alpha$ absorption-line systems (DLAs) that are observed in quasar spectra arise in neutral-gas-rich regions of intervening galaxies. With the highest neutral hydrogen column densities observed $\left(N(H I) \geqslant 2 \times 10^{20}\right.$ atoms $\left.\mathrm{cm}^{-2}\right)$, they are known to trace the bulk of the neutral gas content of the Universe, and are thus powerful probes of galaxy formation and evolution. However, DLAs are extremely rare, and since the Lyman- $\alpha$ line falls in the UV for redshifts $z<1.65$, not many are known at low redshift due to the limited availability of space data. Our HST surveys for DLAs in strong MgII absorbers have been successful at showing that MgII can be used as an unbiased tracer of DLAs. We present new results on their incidence, or redshift number density, $\mathrm{dn} / \mathrm{dz}$, and cosmological neutral gas mass density, $\Omega_{D L A}$, at redshifts $0.11 \leqslant z \leqslant 1.65$, and incorporate results from higher and lower redshift studies in the literature to derive the evolution of neutral gas in the Universe.
\end{abstract}

Keywords. galaxies:evolution, (galaxies:) quasars: absorption lines

Figure 1 summarizes the work presented here (Rao et al. 2016, in prep.). Data from our HST ACS-Prism survey for DLAs (Turnshek et al. 2015), a COS MgII-DLA survey, and GALEX archival spectra were used to update the results of Rao et al. (2006), nearly doubling the sample of low-redshift DLAs found in MgII systems. Fits to the data points are shown, which have the form $d n / d z=(0.025 \pm 0.003)(1+z)^{(1.75 \pm 0.11)}$ and $\Omega_{D L A}(z)=$ $(4.46 \pm 0.30) \times 10^{-4}(1+z)^{(0.68 \pm 0.06)}$.
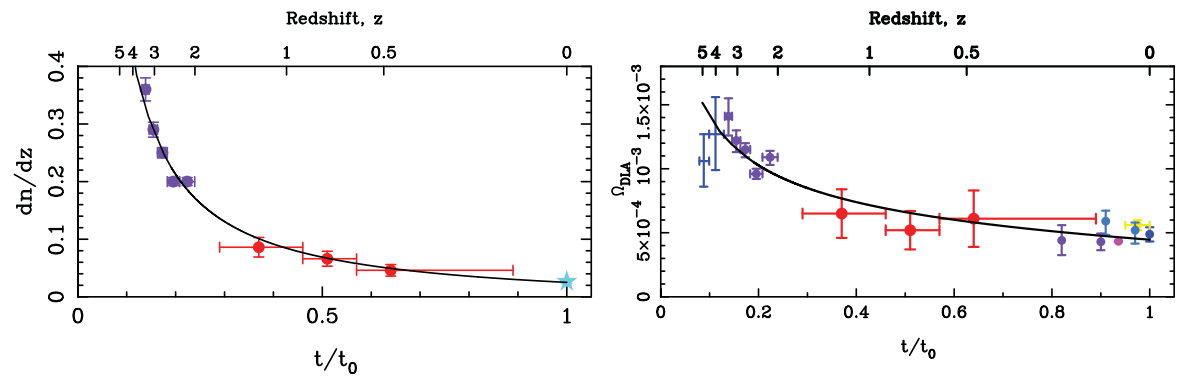

Figure 1. Left: The incidence of DLAs, $\mathrm{dn} / \mathrm{dz}$, and Right: the cosmological neutral gas mass density, $\Omega_{D L A}$, plotted as a function of cosmic time. The present epoch is $t=t_{0}$. Redshift is shown along the top axis. The red circles at $0.11 \leqslant z \leqslant 1.65$ are our new data points. Higher and lower redshift data are from the literature. See Rao et al. (2016, in prep.) for details.

\section{References}

Rao, S., Turnshek, D., \& Nestor, D. 2006, ApJ, 636, 4610

Turnshek, D., et al. 2015, MNRAS, 449, 1536 